\title{
Transatlantica
}

Revue d'études américaines. American Studies Journal

\section{A propos de deux expositions au Kunsthal, Rotterdam}

« Modern Life. Edward Hopper and His Time » et Jacob Holdt « United States 1970-1975»

Jean Kempf

\section{(2) OpenEdition}

Journals

Édition électronique

URL : https://journals.openedition.org/transatlantica/4620

DOI : $10.4000 /$ transatlantica. 4620

ISSN : 1765-2766

Éditeur

Association française d'Etudes Américaines (AFEA)

Référence électronique

Jean Kempf, «A propos de deux expositions au Kunsthal, Rotterdam », Transatlantica [En ligne], 2 |

2009, mis en ligne le 01 février 2010, consulté le 31 janvier 2023. URL : http://

journals.openedition.org/transatlantica/4620; DOI : https://doi.org/10.4000/transatlantica.4620

Ce document a été généré automatiquement le 31 janvier 2023.

\section{(c) (†) $\odot$}

Creative Commons - Attribution - Pas d'Utilisation Commerciale - Pas de Modification 4.0 International - CC BY-NC-ND 4.0

https://creativecommons.org/licenses/by-nc-nd/4.0/ 


\title{
A propos de deux expositions au Kunsthal, Rotterdam
}

\author{
« Modern Life. Edward Hopper and His Time » et Jacob Holdt « United \\ States $1970-1975 »^{1}$
}

Jean Kempf

1 Le musée d'art moderne de Rotterdam, le Kunsthal, situé au cœur d'un ensemble culturel en pleine restructuration qui confirme le rôle de la ville comme métropole des arts, présente une curieuse juxtaposition de deux expositions d'images sur les EtatsUnis. La première, « Modern Life. Edward Hopper and His Time », est une présentation de certains aspects des arts plastiques aux Etats-Unis dans les années 20 et 30 autour de onze toiles de Hopper, l'autre une série de photographies couleur du danois Jacob Holdt faites dans les années 1970-1975, « United States 1970-1975 »².

«Modern Life», organisée par le Whitney Museum qui est un peu le cœur du monde hopperien, utilise la notoriété du peintre pour attirer des visiteurs vers un ensemble de toiles, de photographies, de dessins et de gravures qui ne manque pas d'intérêt. Certes le connaisseur de l'art américain n'y fera pas grande découverte tant au niveau des œuvres que des concepts, mais on peut penser que le visiteur «moyen » attiré par le nom du maître repartira avec une meilleure compréhension de qui il fut (et accessoirement relativisera son admiration pour lui) mais aussi avec une certaine appréciation de la diversité et de la force de l'art américain durant ces deux décennies, un art, il faut bien le dire, assez peu connu en Europe malgré quelques belles expositions récentes ${ }^{3}$.

3 Bien montée dans une scénographie simple et efficace, cette exposition se veut en effet didactique. Autour d'une douzaine de toiles de Hopper installée dans une agora centrale ont été disposées une série de salles chacune organisée autour d'un thème formel illustré par quelques artistes et que le visiteur peut parcourir à sa guise, tout en gardant un œil sur la totalité de l'exposition grâce aux larges circulations entre les espaces. C'est donc bien comme introduction à l'art américain qu'il faut voir cette exposition et non comme présentant un point de vue sur Edward Hopper. Il manquera en effet au spécialiste la dimension historique et génétique de l'œuvre de Hopper car ce 
n'est en rien une exposition sur les sources hopperiennes, ni même un «Hopper \& friends » qui aurait conduit à présenter l'affiche, l'illustration commerciale et d'une manière générale l'image de masse dont on sait pourtant que Hopper les appréciait.

4 Du coup, l'ensemble donne à voir des Etats-Unis plus modernistes que simplement modernes, avec un choix de toiles valorisant l'expérimentation d'un Hartley, d'une O'Keeffe ou d'un Davis. Les « régionalistes » ou les «précisionnistes » sont pourtant là aussi, apportant à celui qui saura bien les regarder un contre-modèle à un Hopper dont on appréciera du coup la subtilité magique du réalisme, un aspect qui a sans nul doute beaucoup compté dans son immense succès public. Enfin, les quelques toiles de la Ash Can School prennent dans cet environnement un relief particulier par le dialogue qui s'établit avec la profondeur psychologique de Hopper cette fois ${ }^{4}$.

5 A côté du plaisir de voir rassemblées en un même lieu des toiles que l'on ne voit que rarement malgré leur célébrité et de découvrir - c'est un bonheur chaque fois renouvelé - la richesse du colorisme américain, la plus belle surprise de l'exposition se trouve dans l'ensemble de dessins et d'aquarelles présenté dans la dernière salle. Trois belles gravures de Hopper (dont la célèbre Night Shadows, 1921), mais aussi des études ou des aquarelles de Demuth, de Burchfield ou de Marin, plus « légères » que certaines de leurs huiles mais aussi plus vivantes dans leur vigueur spontanée, bref des œuvres qui nous rappellent un aspect fort intéressant, mais souvent négligé, de la production américaine.

6 Présentées aux mêmes dates et à l'étage au-dessus, quelques-unes des 15.000 images couleur prises par Jacob Holdt lors de ses cinq années de voyage en auto-stop dans l'Amérique de la première moitié des années 1970. Publiées d'abord dans un ouvrage militant American Pictures $(1977,1985)^{5}$, ces photos font aujourd'hui un retour grâce à l'association GwinZegal de Plouha en Bretagne qui a monté cet ensemble et l'a fait circuler en Europe ${ }^{6}$. Jacob Holdt est un petit phénomène médiatique qui vit depuis presque quarante ans du succès d'un voyage qu'il fit dans l'Amérique des bas-fonds avec, dit la légende, 40 dollars en poche et en donnant son plasma deux fois par semaine pour acheter sa pellicule. Le livre avait fait sensation en Europe à l'époque et, comme souvent dans ces cas-là, était devenu un objet de la propagande anti-américaine lancée par le KGB contre Carter, dans ces moments difficiles de la détente. Le ton de Jacob Holdt se réfère très directement à son prédécesseur danois, Jacob Riis, et mélange un style direct avec une rhétorique héritée du calvinisme (Holdt est fils de pasteur) qui dénonce l'hypocrisie sociale. Devenu d'abord conférencier militant de la cause des démunis américains, il connaît ensuite divers déboires qui l'éloignent du devant de la scène mais il continue à photographier dans le tiers-monde dans les années 1990, tout en participant à des actions humanitaires. Vers le milieu des années 2000, il fait un retour à la faveur du renouveau d'intérêt pour la photographie sociale en période de crise. Mais il faut aussi compter avec un contexte de réception favorable: Michael Moore est passé par là, la dénonciation des Etats-Unis, voire l'anti-américanisme, qui n'aurait pas dépareillé au plus fort de le Guerre froide, sont de retour, sans oublier les réalités et les mythes attachés aux deux derniers présidents, George W. Bush et Barack Obama.

7 Ceux qui pensent, parfois à juste titre, que l'Amérique est le pays de la souffrance sociale, y trouveront ample confirmation de leurs idées. Car le moins que l'on puisse dire est que Holdt ne fait pas dans la dentelle, ni dans les images, ni dans l'accrochage. Tout est brut ici: le montage sans marge de tirages $40 \times 60$ aux couleurs simples et 
criardes (comme peuvent l'être des agrandissements de diapositives 18x24 prises avec un Dial 35-2), les scènes d'intérieur le plus souvent éclairées par un flash rudimentaire. Comme si les images ne se suffisaient pas à elles-mêmes, deux carrousels projettent un montage d'images de l'exposition montrées côte-à-côte dans un jeu de juxtaposition dont le but est évidemment, soit par l'accumulation, soit par le contraste, d'asséner le message simple de Holdt sur l'Amérique des inégalités (le catalogue de l'exposition est, de ce point de vue, beaucoup moins brutal). Car ces images ne sont pas un reportage même empathique - sur les Etats-Unis. C'est du Michael Moore photographique, un pamphlet qui ne retient aucun de ses coups contre une société de l'abondance (le trait le plus saillant des années 1970), une démocratie politique de façade qui tolère en son sein une telle misère et pratique au quotidien la violence essentiellement axée sur des rapports de race ${ }^{7}$.

Holdt s'inscrit en cela idéologiquement et esthétiquement dans une longue tradition de critique sociale, nullement mieux représentée que chez les Américains eux-mêmes (pensons par exemple à Dorothea Lange ou Ben Shahn), et qui ne peut être que favorablement reçu par le public européen d'aujourd'hui.

Mais à les regarder avec le bénéfice de la distance de ces quarante dernières années, on constate combien le petit nombre de marqueurs temporels ("War Widows for Nixon ", la présence du jeune Jay Rockefeller en campagne, un portrait de Ronald Reagan) et leur caractère pour l'essentiel privé en font des images contemporaines. Ce n'est pas de l'Amérique des années 1970 dont on nous parle ici, et preuve en est le succès de Holdt dans ses conférences depuis cette époque. C'est d'une autre Amérique, tout aussi "éternelle », utopique ou mythique que celle du Rêve américain, et qui n'en serait que le versant distopique. On peut trouver le propos simpliste, racoleur, voire faux, mais son efficacité méthodique est certaine. Cette nouvelle présentation des travaux de Holdt est en outre l'occasion d'une redécouverte de ses qualités de photographe.

Alors que l'usage d'images d'appareil amateur par des professionnels est devenue plus courante et que le style «brut» ne choque plus, on perçoit mieux aujourd'hui que Holdt a un sens aigu de la composition qui vaut largement celui de Lee Friedlander ou de Robert Frank, voire du Walker Evans des photos de rue et de Shahn. Son rapport au sujet, son sens de la présence et de la distance ne relèvent pas d'un simple intérêt pour le vernaculaire mais bien d'une compréhension profonde des situations, démontrant une puissance du regard comme pouvait l'avoir Diane Arbus. Cela fait de ces « images américaines " un ensemble passionnant qui nous permet aussi de revisiter de larges pans de la photographie américaine, de la FSA à Winogrand en passant par Weegee, ainsi que d'autres figures plus contemporaines (et donc «héritières» de son travail) comme Larry Fink et Nan Goldin.

BIBLIOGRAPHIE 


\section{Catalogues}

Jacob Holdt: United States, 1970-1975. Göttingen: Steidl; Saint-Brieuc: Gwin Zegal, 2007.

Modern Life. Edward Hopper en zijn tijd. Munich: Hirmer; Zwolle: Wanders Uitgevers, 2009.

\section{NOTES}

1. «Modern Life. Edward Hopper and His Time» du 26 septembre 2009 au 17 janvier 2010, « United States 1970-1975 » du 19 septembre 2009 au 10 janvier 2010. « Modern Life » a aussi été présentée à Hambourg au Bucerius Kunst Forum du 9 mai au 30 août 2009.

2. «Modern Life. Edward Hopper and His Time» du 26 septembre 2009 au 17 janvier 2010, « United States 1970-1975 » du 19 septembre 2009 au 10 janvier 2010. « Modern Life » a aussi été présentée à Hambourg au Bucerius Kunst Forum du 9 mai au 30 août 2009.

3. La quasi totalité des œuvres se situe dans cette période.

4. On voit ici bien l'étrangeté de la composition et des couleurs de Robert Henri qui lui donne un caractère presque fauviste.

5. Jacob Holdt, American Pictures: A Personal Journey Through the American Underclass (Copenhague: American Pictures Foundation, 1985). Edition originale : Copenhague : Information, 1977.

6. Museum Folkwang, Essen, 17 mars-3 juin 2007, Photoforum Pasquart, Bienne, 24 juin-26 août 2007, Langhans galerie, Prague, 21 novembre 2007-20 janvier 2008, Centre national de l'audiovisuel, Luxembourg, 21 mars-15 mai 2008, Musée Nicéphore Niépce, Chalon-sur-Saône, 2008.

7. On voit déjà dans American Pictures se profiler une thématique que Holdt a approfondie depuis, celle des White Supremacists.

INDEX

Thèmes : Trans'Arts

\section{AUTEUR}

\section{JEAN KEMPF}

Université Lyon 2 